\title{
A PROPOS D'UNE NOUVELLE ESPÈCE \\ DE NEMATOPARATAENIA (NEMATOPARATAENIA BRABANTIAE N. SP.) DU CYGNE, DÉCRITE POUR LA PREMIÈRE FOIS EN BELGIQUE
}

\author{
Par Christiane cotTEleER et Paul schyNS
}

En janvier 1961, nous avons reçu à l'Institut le cadavre d'un cygne provenant de Chaudfontaine (province de Liége) mais originaire de Grimbergen, situé dans la province de Brabant.

L'animal, en état d'amaigrissement fort prononcé, a été autopsié ; l'examen a révélé une nette décoloration du foie et des reins, ainsi qu'une entérite légèrement hémorragique.

Les ensemencements de pulpe d'organes sur les milieux de culture habituels n'ont donné lieu à aucun développement.

Dans l'intestin, particulièrement dans la partie antérieure de celui-ci (duodénum et jéjunum), nous avons découvert une grande quantité d'Helminthes. Ceux-ci se trouvaient groupés en pelotes de 15-20 sujets tous les $5-10 \mathrm{~cm}$.

L'examen de ce matériel parasitologique démontra qu'il s'agissait de Cestodes. En effet, bien que le corps soit cylindrique et sans segmentation aucune, le parasite est pourvu d'un énorme rostre et de quatre ventouses. La présence d'un seul sillon profond longeant latéralement le corps du parasite et l'absence totale d'orifices sexuels l'ont fait classer dans l'ordre des Aporidæ (Fuhrmann, 1933), famille des Nematoparataenidx.

Comparant les dimensions données par Fuhrmann (1) dans la description du Nematoparataenia southwelli découvert au Lac Takern en Suède et celles du parasite se trouvant en notre présence, nous nous sommes rendu compte que nous étions en possession d'une nouvelle espèce, à laquelle le nom de Nematoparataenia brabantix n. sp. a été donné.

\section{Description}

Si le Nematoparataenia southwelli est de très petite taille (1,6 à 2,5 mm.), et le premier Nematoparataenia, décrit par Maplestone et Southwell (2), sous le nom de Nematoparataenia paradoxa, est d'une 
taille supérieure $(9 \mathrm{~mm}$.), le nôtre, quoique étroitement apparenté aux deux espèces décrites, se trouve dans une position intermédiaire, ayant une longueur moyenne de $4,4 \mathrm{~mm}$., les exemplaires retrouvés allant de 4 à $6 \mathrm{~mm}$.

Le corps du Cestode, de forme cylindrique, a un diamètre variant de 250 à $355 \mu$, donc légèrement plus étroit que Nematoparataenia southwelli $(300-370 \mu)$, ce qui, dans l'ensemble, lui donne un aspect moins ramassé, plus grêle.

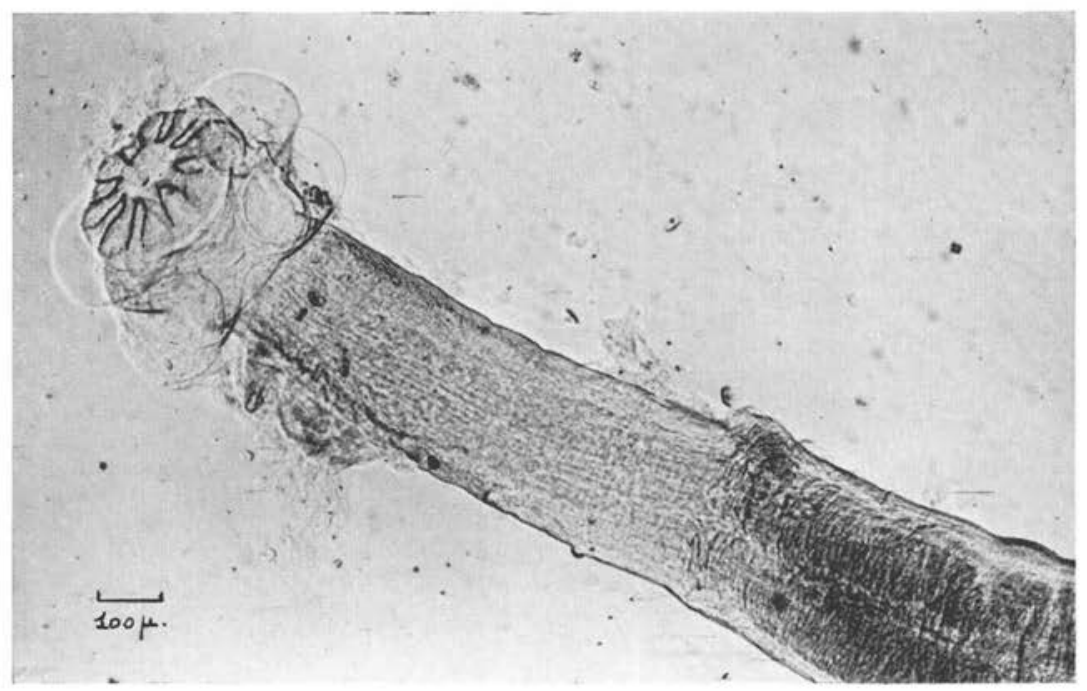

FIG. 1. - Nematoparataenia brabantia, n. sp. : scolex et zone antérieure

Le scolex (fig. 1), énorme, est beaucoup plus large que long, son diamètre étant de 425 à $525 \mu$, pour une longueur de $225-250 \mu$.

Le rostre (fig. 2 et 3 ), volumineux, très musculeux et toujours dévaginé sur les exemplaires étudiés, a la forme d'un entonnoir ou d'un calice renversé dont le sommet arrondi repose sur la base du cou. L'extrémité supérieure du rostre est formée par une rosace de huit profondes ondulations bordées d'une seule rangée de très petits crochets. Nous avons dénombré 80 à 90 crochets par boucle, soit 640 à 720 crochets pour la totalité de la ligne ondulée. Les crochets, très fins, mesurent 6 à $8 \mu$.

Le rostre est entouré de quatre grandes ventouses, proéminentes, à ouverture dirigée vers le haut, ayant une longueur de $160 \mu$ et une largeur de $175 \mu$.

Ainsi qu'il a été dit plus haut, le corps du Cestode ne présente 


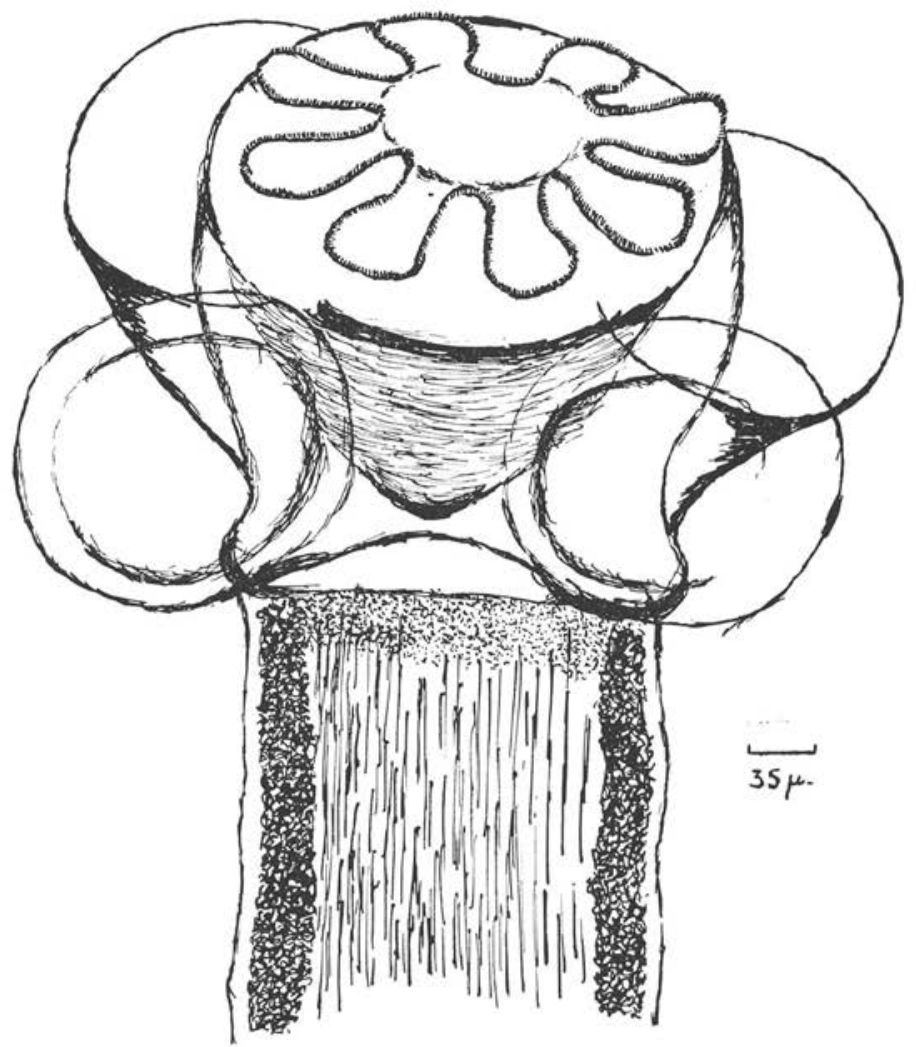

Fis. 2. - Nematoparalaenia brabantia n. sp. : rostre et ventouses

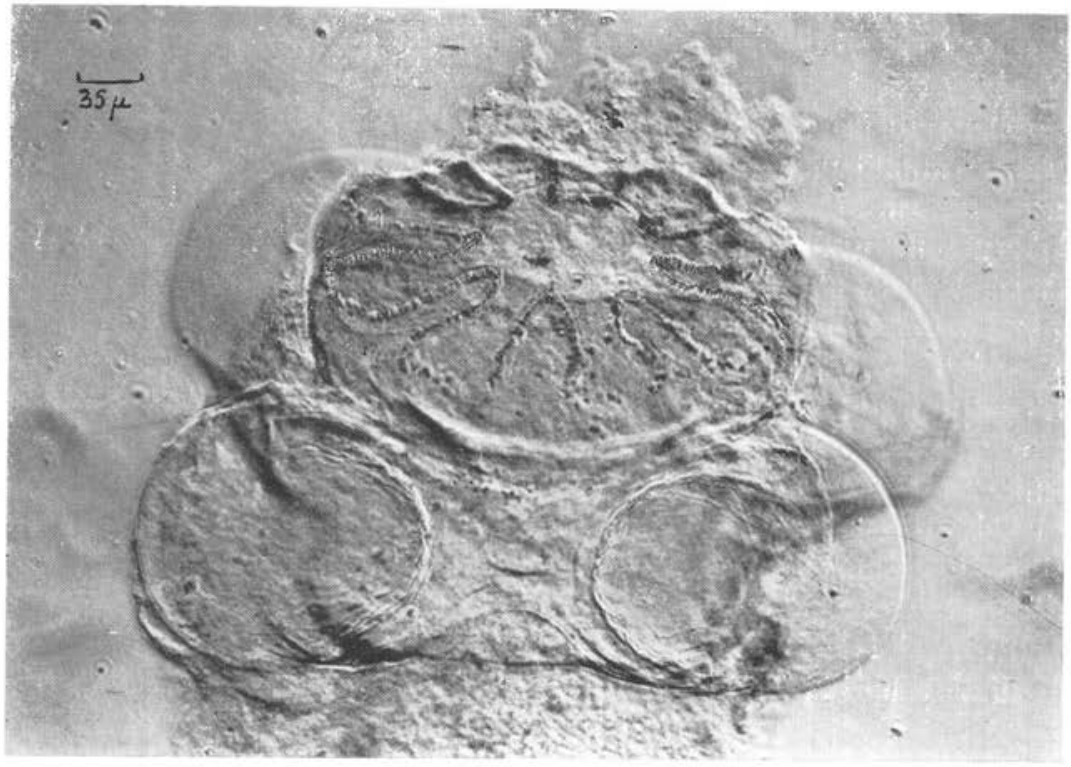

Fig. 3. - Scolex de Nematoparataenia brabantiæ n. sp. 
aucune segmentation ; d'autre part, nous n'avons pu mettre en évidence ni ouvertures extérieures, ni conduits sexuels mâles ou femelles ; quant au sillon latéral ayant l'aspect d'une profonde dépression, il est nettement visible dans le tiers postérieur du parasite.

La structure intérieure du corps est d'une texture des plus difficiles à déterminer ; nous y avons toutefois distingué quatre zones (fig. 4), réparties comme suit et dont les dimensions correspondent pour un adulte de $4,5 \mathrm{~mm}$. :

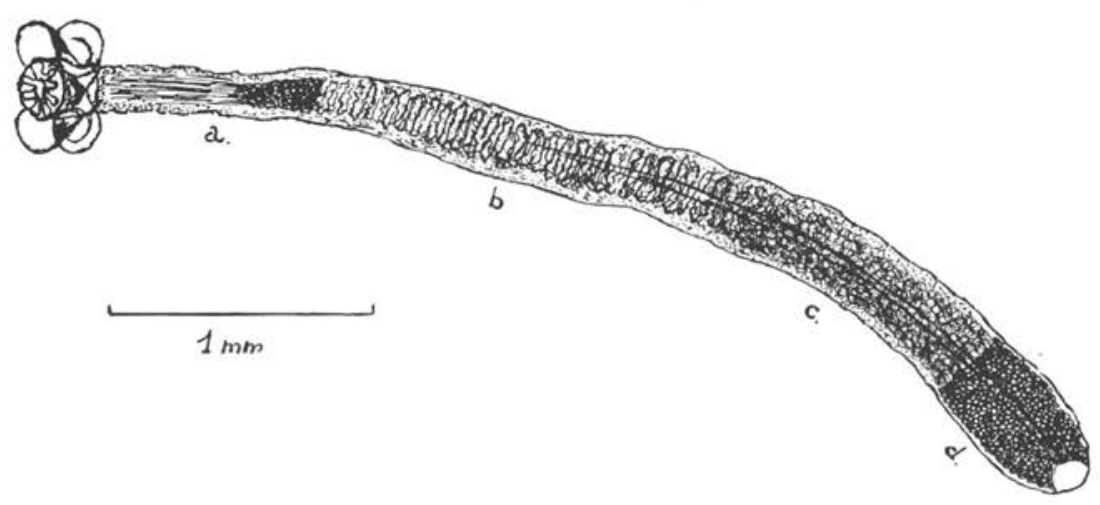

Fig. 4. - Nematoparataenia brabantia n. sp.

$a=$ zone antérieure
$b=$ zone de follicules testiculaires
$c=$ zone de follicules ovariens
$d=$ zone ovigère

1) une portion antérieure (fig. $4, a$ ), où l'on distingue un cou court et très musculeux, suivie d'une zone à fibres longitudinales sur une longueur d'environ un demi-millimètre et se terminant par une zone de cellules non différenciées, très petites et fort serrées ;

2) une zone de follicules testiculaires (fig. 4, b), de formes assez irrégulières, ayant vaguement la conformation de canaux très sinueux à contours imprécis et s'étendant sur une portion de $1,5 \mathrm{~mm}$. ;

3) une zone d'un millimètre de follicules ovariens (fig. 4, c), formée d'un très grand nombre de très petits sacs folliculaires de forme ovalaire ;

4) une zone ovigère de $0,5 \mathrm{~mm}$. de long (fig. $4, d$ et fig. 5), véritable poche à œufs mûrs, excessivement nombreux ; c'est d'ailleurs la seule portion du corps qui soit bien nette et distincte. Les œufs, for- 
més d'une double enveloppe, mesurent 20 sur $16 \mu$ et contiennent six erochets.

Le cycle biologique du Cestode, ainsi que son action pathogène restent à déterminer.

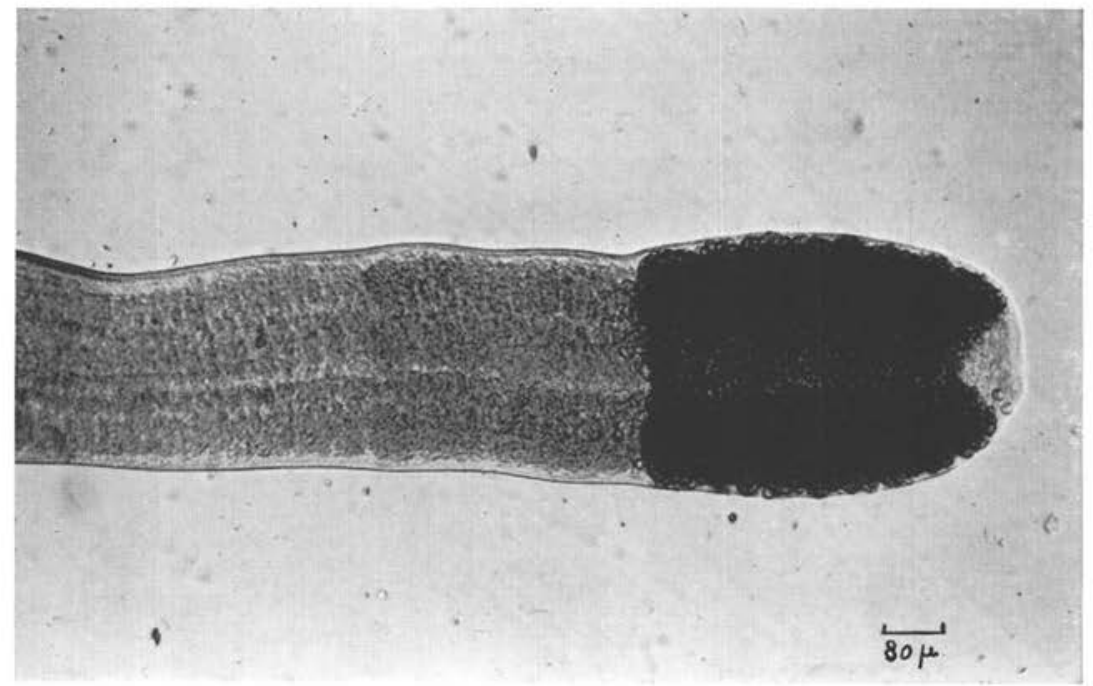

Fig. 5. - Nematoparataenia brabantia n. sp. : zone ovigère

Le fait suivant est toutefois à signaler : considérant d'une part les lésions observées à l'autopsie et, d'autre part, les analyses bactériologiques restées négatives, ainsi que l'absence totale d'autres parasites, l'on est en droit de conclure que l'action pathogène de Nematoparataenia brabantiz est loin d'être négligeable.

D'autre part, l'occasion de retrouver, une quinzaine de jours après la découverte de Nematoparataenia brabantiæ, un autre cygne porteur du même parasite, mais provenant d'une autre région, laisse supposer une certaine fréquence de cette téniase en Belgique.

Grâce à une stricte surveillance et à une vigilance accrue, nous espérons pouvoir donner d'ici peu une réponse exacte quant à la pathogénité et à l'existence éventuelle de foyers à Nematoparataenia. 


\section{RÉSUMÉ}

La découverte en Belgique d'une nouvelle et troisième espèce de Nematoparataenia, le Nematoparataenia brabantiæ n. sp., du cygne, ainsi que sa description, sont données.

Institut National de Recherches Vétérinaires, Uccle (Bruxelles)

\section{Bibliographie}

1. Fuhrmann (O.), 1933. - Un Cestode aberrant. Bull. Soc. Neuchâteloise des Sc. Nat., 58, 107-120.

2. Maplestone (P. A) et Southwell (T.), 1922. - Notes on Australiae Cestodes. V. Three Cestodes from the black swan. Ann. Trop. Med. \& Paras., 16, 189-193. 